\title{
Analysis of Multicast Routing Protocols in Wireless Mesh Networks
}

\author{
${ }^{1}$ Mohammad Masdari, ${ }^{2}$ Reza Khorsandi, ${ }^{3}$ Parinaz Aghapoor \\ ${ }^{1}$ Department of Computer Engineering, Urmia Branch, Islamic Azad University, Urmia, Iran, \\ 2Department of Computer Engineering, Science and research Urmia Branch, Islamic Azad University, Urmia, \\ Iran, \\ ${ }^{3}$ Department of Computer Engineering, Science and research Urmia Branch, Islamic Azad University, Urmia, \\ Iran.
}

\begin{abstract}
Wireless mesh networks (WMNs) are becoming increasingly popular as they have significant advantages over competing technologies. In WMNs, routing algorithms are classified into various categories such as unicast, multicast, and multipath routing algorithms. Due to this classification, we select multicast category to be reviewed in our work. A variety of multicast routing solutions have been proposed in the literature. The aim of this survey is to present taxonomy of routing protocols for wireless mesh networks by illustrating the main features and discussing the key challenges.
\end{abstract}

\section{Introduction}

Wireless mesh networks (WMNs) have emerged as an evolved network technology to provide better services and cost effective solutions to the users. In typical wireless mesh network, there are two kinds of nodes, i.e., mesh routers(MR) and mesh clients(MC) which are shown in figure $1[1,2,3]$. The mesh routers form the backbone of the WMNs and provide network access for the mobile clients [2,3]. Each mesh router operates not only as an access point but also as a relay node that can forward packets to other mesh routers according to the routing information [2]. Mesh routers are stationary with power supply otherwise clients which may be mobile or stationary with limited power or ability. These networks have properties like dynamically self-organized, self-configured and self-healing that come into the advantages of easy deployment and maintenance, high reliability, and large coverage[2- 4].

There are some critical performance factors for WMNs on network design and application as follow.

\footnotetext{
- Radio Techniques: Many approaches have been proposed to increase capacity and flexibility of wireless systems in recent years [5-8]. Typical examples include directional and smart antennas $[9,10]$ multiple input multiple output (MIMO) systems [11, 12], and multiradio/multi-channel systems $[13,14]$. To further improve the performance of a wireless radio and control by higher layer protocols, more advanced radio technologies such as reconfigurable radios, frequency agile/cognitive radios $[15,16]$, and even software radios [17] have been used in wireless communication. These advanced wireless radio technologies that are in infancy all require a revolutionary design in higher- layer protocols, especially MAC and routing protocols.
}

- Scalability: Scalability is the ability of the protocol to scale well in a network with a large with no node [10]. To ensure the scalability in WMNs, all protocols from the MAC layer to the application layer need to be scalable [7].

- Quality of Service (QoS): In addition to being supported by the medium access control (MAC) layer and the forwarding engine, selecting the "best" routes for different traffic[18], QoS support is essential for supporting time critical traffic sessions [19].

- Mesh connectivity: Mesh connectivity should be done in such a way that it could self-organize problems or errors [20]. The MAC and the routing protocol should have knowledge of the network topology as they have to route data from one point to another.

- Security: Without a convincing security solution, WMNs will not be able to succeed due to lack of incentives by customers to subscribe to reliable services [2].

Routing involves two basic activities: determining optimal routing paths and transporting information groups (typically called packets) through an internetwork. Routing protocol specifies how routers report changes and share information with other routers in the network that they can reach [21]. For self-organized networks, the protocols are expected to propose some functions providing management, constructing and selecting routes, maximizing the capacity of the network and minimizing the packet delivery delays $[5,22]$. The classification that has been suggested for these protocols includes unicast, broadcast and multicast protocols.

The first category or Unicast is a communication from one source to one receiver which the source and receiver may be on the same or on different networks. The second one or multicasting is a fundamental routing service in wireless mesh networks due to its numerous potential applications such as video conferencing, online games, and webcast [23]. It is a one-to-many communication form that sends data from a source node to a set of destination nodes. A multicast group contains a special host which is responsible for transmitting data packets to other hosts in the same group [24]. To facilitate communication within a group of users, these protocols are used to discover routes between nodes [25]. The multicast router will periodically send an IGMP (Internet Group Management Protocol) membership query to all hosts on directly attached network to determine the list of members 
of active membership group. Three main classification presented for multicast protocols are proactive (distance vector or link-state type), reactive (also referred to as ondemand) and hybrid (combination of reactive and proactive). According to the classification, some multicast protocols that are applied include ODMRP, MAODV which uses reactive methods, MOSPF that uses proactive methods, and MSR which uses hybrid method [26].

In this paper, we focus on the multicast routing protocols in wireless mesh networks and present a classification for these protocols. The rest part of the paper is organized as follows. We present classification of multicast routing solutions in section 2 and briefly discuss about each multicast routing scheme. Then in section 3, we provide concluding remarks.

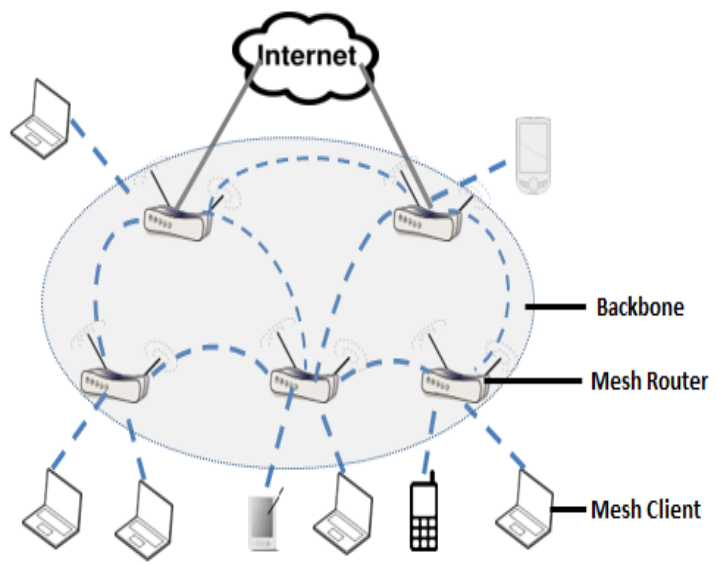

Figure 1: Wireless mesh network.

\section{Classification of Multicast Routing Protocols}

Multicast protocols organized in groups by establishing dissemination structures such as trees or meshes, dynamically updated as nodes join or leave the group [27]. Developing a multicast routing protocols, there are many characteristics and challenges such as the dynamisms of the network topology, the constraints of energy, limitation of network scalability, and the different characteristics between wireless links and wired links such as limited bandwidth and poor security [28, 29] should be taken into consideration. As figure 2 exhibits, existing multicast routing approaches can be divided into three protocols as tree-based multicast protocols, mesh-based multicast protocols and hybrid multicast protocols.

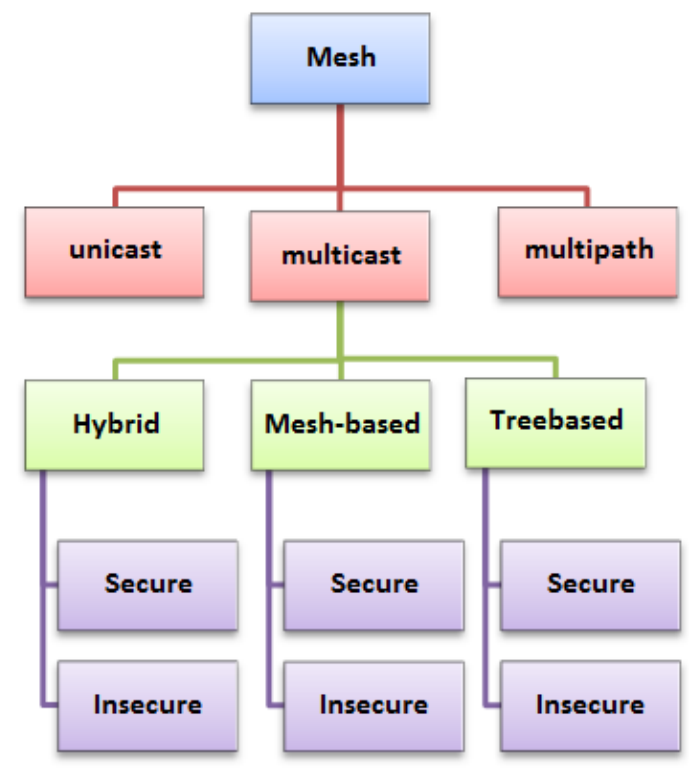

Figure 2: Taxonomy of routing protocols for wireless mesh networks

\subsection{Tree-Based Multicast Routing Protocol}

A tree-based multicast routing protocol establishes and maintains a shared multicast routing tree to deliver data from a source to receivers of a multicast group [56]. The key idea in a tree-based algorithm is that most nodes require storage only for a few children, instead of all possible ones. Such algorithms, therefore, make frugal use of memory at the expense of doing more memory lookups [54].Since the structure of tree-based multi- casting is reconfigured frequently in dynamic networks, it may be highly unstable. Examples of tree-based multicast routing protocols can be Multicast Adhoc On-demand Distance Vector routing protocol (MAODV), and Adaptive Demand-driven Multicast Routing protocol (ADMR).

\subsubsection{Multicast Ad Hoc On-demand Distance Vector Protocol}

As mentioned before, a good illustration for tree-based multicasting is Multicast Ad Hoc On-Demand Distance Vector (MAODV). This protocol [30- 32] is the multicast extension of AODV [33] which is used for unicast traffic [34]. Here the route discovery is based on a route request RREQ and route reply RREP cycle as shown in Fig. 3. When MAODV discovers multicast routers it originates a route request (RREQ) message. Then the members of multicast group respond to the route reply message [35]. If a node is non-member of multicast group and receives a route RREQ, it rebroadcasts RREQ to its neighbors. But if the RREQ is not joining request, any node of the multicast group may respond [36].

MAODV always choose the shortest path (tree-based) and since it unicasts the reply, it has low overhead. In spite of these advantages, it has some drawbacks e.g.it is shared-tree and there is no backup path of link if the link is broken. Furthermore, a high percentage of bad frames in video transmission are may occur because of packet loss (link broken). Some multiple interruptions may also be seen in video watched. 

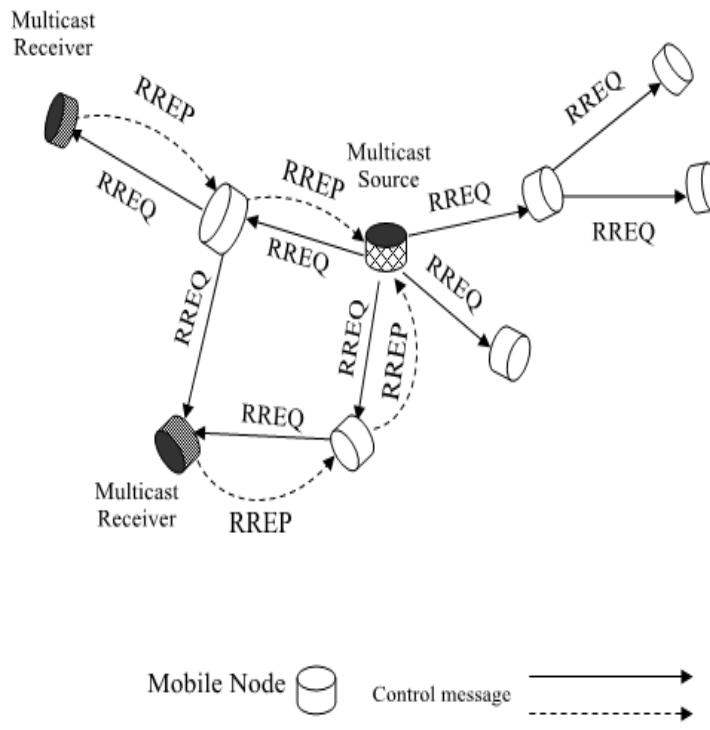

Figure3: MAODVR outing Protocol

\subsubsection{Adaptive Demand-driven Multicast Routing protocol ((ADMR)}

J.G. Jetcheva et al [58], introduce Adaptive Demand-Driven Multicast Routing protocol (ADMR), a new on-demand ad hoc network multicast routing protocol that attempts to reduce as much as possible any non-on-demand components within the protocol. The use of on-demand techniques in routing protocols has significant advantages in terms of reducing the routing protocol's overhead and improving its ability to react quickly to topology changes in the network. Multicast routing state is dynamically established and maintained only for active groups and only in nodes located between multicast senders and receivers. Each multicast data packet is forwarded along the shortest-delay path with multicast forwarding state, from the sender to the receivers, and receivers dynamically adapt to the sending pattern of senders in order to efficiently balance overhead and maintenance of the multicast routing state as nodes in the network move or as wireless transmission conditions in the network change. To summarize, the novel features of ADMR include:

ADMR uses no periodic network-wide floods of control packets, periodic neighbor sensing, or periodic routing table exchanges, and requires no core. The protocol adapts its behavior based on application sending pattern, allowing efficient detection of link breaks and expiration of routing state that is no longer needed. ADMR handles bursty sources by sending limited keep-alives for a period, to distinguish disconnections from lack of data. If there are no receivers in the network, sources only flood existing data at infrequent intervals (to heal partitions) and do not transmit other data or control packets. Furthermore, this is optional functionality and does not affect the main routing mechanisms used by the protocol.

\subsection{Mesh-Based Multicast Routing Protocol}

Mesh-based multicast routing protocol sustains a mesh consisting of a connected component of the network containing all the receivers of a group [55, 57]. Two wellknown examples of mesh-based multicast routing approaches are Core Assisted Mesh protocol (CAMP) and On-Demand Multicast Routing Protocol (ODMRP).

\subsubsection{On-demand Multicast Routing Protocols}

On-Demand Multicast Routing Protocol (ODMRP)[37, 38] is a mesh-based example that can make use of unicast technique to send multicast data packet from the sender nodes toward the receivers in the multicasting group [35]. The group membership [35] is established and maintained in ODMRP by the source.

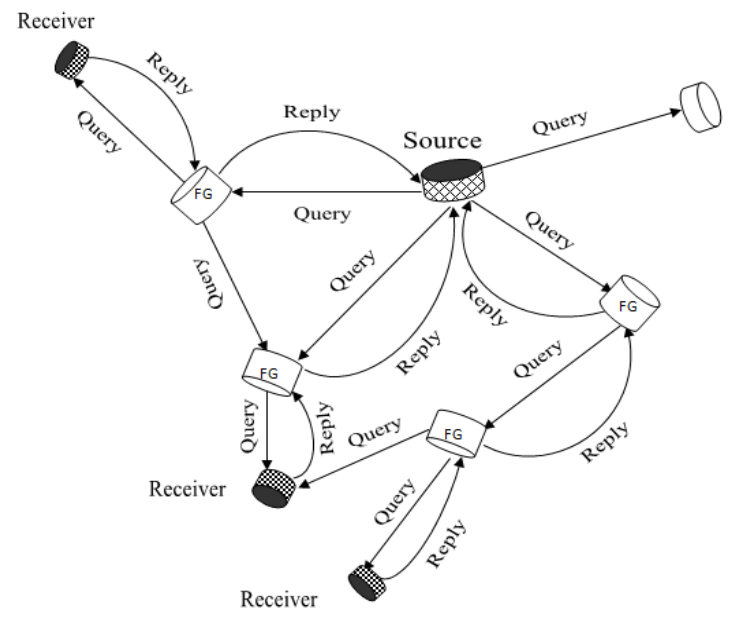

Figure4: Mesh creation in ODMRP

If node wishes to send a packet but has no rout to that group it broadcasts a JOIN QUERY (JQ) message to the entire network which is shown in figure 4 . When a node receives a non-duplicates JOIN QUERY message, it records the address and rebroadcasts the message. As the message reaches a multicast receiver, the receiver creates or updates the source entry in its multicast group and creates a JOIN REPLY packet that is broadcasted to its neighbors. Receives a JOIN REPLY message, the node knows whether it is on the path to the source or not. If it does, it realizes that the path becomes a part of the forwarding group (FG) for that source and broadcast it's JOIN REPLY. Despite, membership and route information is updated by periodically (certain interval times) sending JOIN QUERY packet [34] (Fig2). Generally ODMRP has the following advantages:

- No explicit leave message (member nodes refreshed when needed by source).

- Multiple paths to one destination (mesh approach).

- There is a Backup path of link if link is broken.

Also, its disadvantages are:

- Because of broadcasting the reply to many nodes, it has High Overhead.

- Its topology is Complex.

\subsubsection{Core-Assisted Mesh Protocol}

In [59] E.L. Madruga et al, indicate that Most of the multicast routing protocols are based on shared or source-based trees; however, keeping a routing tree connected for the purpose of data forwarding may lead to a substantial network overhead. A different approach to multicast routing consists of building a shared mesh for each multicast group. In multicast meshes, data packets can be accepted from any router, as opposed to trees where data packets are only accepted from routers with whom a "tree branch" has been established. The difference 
among multicast routing protocols based on meshes is in the method used to build these structures. The key difference between a mesh and a tree structure is how data packets are accepted to be processed. A router is allowed to accept unique packets coming from any neighbor in the mesh, as opposed to trees where a router can only take packets coming from routers with whom a tree branch has been established. Therefore, keeping the branch information updated is one extra challenge protocols based on trees have to face in a mobility scenario. Some mesh-based protocols require the flooding of sender or receiver announcements over the whole network. They present Core-Assisted Mesh Protocol (CAMP), which uses meshes for data forwarding, and avoids flooding or control packets by generalizing the notion of core-based trees introduced for internet multicasting. Group members form the mesh of a group by sending join requests to a set of cores.

\subsection{Multicast Open Shortest Path First (MOSPF)}

MOSPF [39] protocol is an improvement multicast OSPF (Open Shortest Path First) protocol which provides efficient critical performance factors for WMN [40].Routers in MOSPF maintain a current image of the whole network topology through the unicast OSPF protocol[41].IGMP (Internet Group Management Protocol) is used in MOSPF routers to examine membership in multicast group by broadcasting IGMP Host Membership Queries and receiving IGMP Host Membership Reports $[39,40]$. Scaling well over large networks just like OSPF is one of the advantages of MOSPF. But it should be noted that every router must maintain the membership information of each group. So it scales poorly if there are many multicast groups [42]. This protocol also requires a lot of processing capacity.

\subsection{Hybrid Multicasting}

It is a type of protocol that has the combination of both treebased and mesh-based multicasting routing protocols. Typical examples include Mesh Networks Scalable Routing and Hybrid Distance Vector routing protocol.

\subsubsection{Mesh Networks Scalable Routing (MSR)}

The Mesh Network company has also developed a proprietary hybrid ad-hoc routing protocol that combines both proactive and reactive routing protocols [43, 44], called Mesh Networks Scalable Routing (MSR) [45]. With this methodology, network topology dynamics, local RF conditions and degree of node mobility influence the routing metrics used on a moment-by-moment basis. It is worth noting that the MSR is used for the Motorola's multi-hop solutions. It Provides scalability with less resource consumption and routs the packets easily by considering the heat of the nodes near to gateways[46]. On the other hand, the External environment heat can affect the gates which Provides gate ways. Unnecessary power consumption during some load balancing may also affect the heat parameter of a node.

\subsubsection{Hybrid Distance Vector (HDV) Routing Protocol}

In [47] A. Le at el, propose a Hybrid Distance Vector (HDV) routing protocol for hybrid mesh networks which combines the tree-based routing strategy with the reactive routing strategy. The proposed HDV protocol is an extension of the AODV routing protocol in a multi-radio environment based on integrating a proactive component into the reactive routing of the AODV. A proactive component builds a tree-topology in the backbone of mesh routers which links all the mesh routers with the gateway node. Meanwhile, the mesh clients reactively discover routes to the gateway which is stored in the routing table of mesh routers. In HDV, a gateway node periodically broadcasts a route request message that only allows mesh routers to establish a route toward the gateway node. As such, these routes are more stable due to the stationary and high capacity of mesh routers. Plus, the latency and routing overhead for discovering route towards a gateway node are reduced for the mesh clients. The low-complexity implementation of the HDV protocol also makes it applicable to large hybrid mesh networks.

\begin{tabular}{|c|l|l|}
\hline \multicolumn{3}{|c|}{ Table 1: Evaluation of Multicast Structures } \\
\hline \multirow{3}{*}{ Tree-based } & \begin{tabular}{l}
\multicolumn{1}{c|}{ Advantages } \\
Minimal data traffic, \\
Bandwidth efficient, \\
High forwarding, \\
Low routing overhead.
\end{tabular} & $\begin{array}{l}\text { Huge control traffic to Maintenance of tree, } \\
\text { Poor scalability, } \\
\text { High possibility of dropping (no backup path } \\
\text { exist) } \\
\text { Vulnerability to high mobility. }\end{array}$ \\
\hline \multirow{5}{*}{ Hesh-based } & $\begin{array}{l}\text { Relative reliability, } \\
\text { Multiple paths (more efficient if a path broke), } \\
\text { Quickly reconfigurable. }\end{array}$ & $\begin{array}{l}\text { Probability of having routing loops, } \\
\text { high overhead because of control packet flooding, } \\
\text { High number of forwarding nodes, } \\
\text { Excessive message overhead (low efficiency). }\end{array}$ \\
\hline $\begin{array}{l}\text { Reduces disadvantages of proactive and reactive } \\
\text { routing protocols, } \\
\text { No route setup latency for short distance connections, } \\
\text { Lower routing overhead due to reactive routing. }\end{array}$ & $\begin{array}{l}\text { Advantage depends on amount of nodes activated, } \\
\text { Reaction to traffic demand depends on gradient of } \\
\text { traffic volume. }\end{array}$ \\
\hline
\end{tabular}




\subsection{Evaluation of Multicast Structures}

The performance of hybrid, mesh and tree based multicast routing has been evaluated and shown in Table1.Since Meshbased algorithms create a structure in which more than only one path can connect the sender with the receiver, they are recognized as the most reliable onesbut have higher

overhead due to redundant retransmissions. In comparison, even though in tree-based algorithms only one path from sender to receiver exists, it makes the routing much more effective and eliminates the possibility of loops occurring in the network, but require more complex operations to create and maintain the multicast tree, and are less resilient to failures. Moreover, both the sender (sender-initiated) and the receiver (receiver-initiated) may initiate creation of the multicast tree. The existing research [48, 49] shows that, where changes in topology are common, mesh-based algorithms show better results than tree-based algorithms, which is due to the existence of redundant links in the mesh structure.

The comparison of multicast mesh routing protocols $\mathrm{s}$ summarized in Table 2.

\begin{tabular}{|c|c|c|c|c|}
\hline \multicolumn{5}{|c|}{ Table 2: Properties of mesh-based multicast routing protocols } \\
\hline Protocol & Scalability & Reliability & Routing metrics & Type \\
\hline ODMRP & Low & High & Shortest path & Reactive \\
\hline MAODV & High & Low & $\begin{array}{c}\text { Shortest path to another multicast } \\
\text { member along the existing shared tree }\end{array}$ & Reactive \\
\hline MOSPF & Low & High & Hop count & Proactive \\
\hline MSR & High & High & ARM(Air time routing metric), Hop count & Hybrid \\
\hline
\end{tabular}

\section{Conclusion}

Four different multicast protocols with the advantages and disadvantages have been discussed in this paper. The protocols were compared with regard to similarities and differences between mesh and tree-based multicast protocols which can be of great help to choose the most appropriate in different situations. It is worth saying again that multicast tree-based routing protocols in spite of being efficient and useful to satisfy scalability issues, have some drawbacks due to mobile nature of nodes that participate in multicast session. On the other hand, mesh-based protocols which provide more robustness against mobility save the large size of control overhead used in tree maintenance. After all, a good solution was found which includes the advantages of both protocols called hybrid multicast. As a matter of fact, it has been supposed that it is really difficult to design a multicast routing protocol considering all the mentioned issues. Still it is a problem for researcher to develop a single protocol which can satisfy as many goals as possible in the future.

\section{References}

[1] H. Chenga, S. Yangb, Joint QoS multicast routing and channel assignment in multiradio multichannel wireless mesh networks using intelligent computational methods, Applied Soft Computing, vol. 11,2011, pp. 1953-1964.

[2] I. F. Akyildiz, X. Wang, W. Wang, Wireless mesh networks: a survey, Computer Networks, vol. 47, 2005, pp. 445-487.

[3] Y. Han, X. Gui, X. Wu, X. Yang, Proxy encryption based secure multicast in wireless mesh networks, Journal of Network and Computer Applications, vol. 34, 2011, pp. 469-477.

[4] A. Y. Al-Dubai, L. Zhao, A. Zomaya, G. Min, A Novel Multicast Gateway Based Multi-hop Routing for Wireless Mesh Networks, IEEE International Conference on Communications, Kyoto, Japan, 2011.

[5] S.D. Odabasi, A.H.Zaim, A Survey on Wireless Mesh Networks, Routing Metrics and Protocols, International Journal Of Electronics, Mechanical and Mechatronics Engineering, vol.2 ,pp.92-104.
[6]Elizabeth M. Royer, Chai-KeongToh, "A Review of Current Routing Protocols for Ad Hoc Mobile Wireless Networks", IEEE Personal Communications, April, 1999.

[7] I. F. Akyildiz, A Survey on Wireless Mesh Networks, IEEE Radio Communications, 2005, pp.523-530.

[8] A.Valarmozhi, M.Subala, V.Muthu, Survey of Wireless Mesh Network, International Journal of Engineering and Innovative Technology (IJEIT) vol. 2, 2012, pp.338-342.

[9] R. Ramanathan, On the performance of ad hoc networks withbeam forming antennas, in: ACM International Symposium on Mobile Ad Hoc Networking and Computing (MOBIHOC), October 2001, pp. 95-105.

[10] S. S.N.Rao, Y. K. S.Krishna, K.N.Rao, A Survey: Routing Protocols for Wireless Mesh Networks, International Journal of Research and Reviews in Wireless Sensor Networks (IJRRWSN), vol. 1, 2011,pp.43-47.

[11] W. Xiang, T. Pratt, X. Wang, A software radio test bedfor two-transmitter two-receiver space time coding wireless LAN, IEEE Communications Magazine, 2004, pp. 20-28.

[12] K. Sundaresan, R. Sivakumar, M.A. Ingram, T.-Y. Chang, A fair medium access control protocol for adhoc networks with MIMO links, in: IEEE Annual Conference on Computer Communications (INFOCOM) , 2004, pp. 2559-2570.

[13] J. So, N. Vaidya, Multi-channel MAC for ad hoc networks: handling multi-channel hidden terminals using a single transceiver, in: ACM International Symposiumon Mobile Ad Hoc Networking and Computing (MOBIHOC), 2004,pp. 222-233.

[14] A. Adya, P. Bahl, J. Padhye, A. Wolman, L. Zhou, A multi-radio unification protocol for IEEE 802.11 wireless networks, in: International Conferences on Broadband Networks (Broad Nets), 2004.

[15] M. McHenry, Frequency Agile Spectrum Access Technologies, FCC Workshop on Cognitive Radios, 2003. 
[16] B. Lane, Cognitive radio technologies in the commercial arena, in: FCC Workshop on Cognitive Radios, 2003.

[17] J. Mitola III, Software Radio Architecture: ObjectOriented Approaches to Wireless System Engineering, Wiley Inter-Science, New York, 2000.

[18] J.Jun, M.L. Sichitiu, MRP: Wireless mesh networks routing protocol, Computer Communications, 2008, pp.1413-1435.

[19] J.Jain, M.Fatima, Dr. R.Gupta, Dr. .K.Bandhopadhyay, Overview And Challenges Of Routing Protocol And Mac Layer In Mobile Ad-hoc Network, Journal of Theoretical and Applied Information Technology,2005-2009.

[20] V.Mynampati，D.Kandula， R.Garimilla，K. Srinivas, Performance and Security of Wireless Mesh Networks, Blekinge Institute of Technology,2009.

[21] S. Sumathy, B.Yuvaraj, E.SriHarsha, Analysis of Multicast Routing Protocols: Puma and Odmrp, International Journal of Modern Engineering Research (IJMER) ,Vol.2,2012, pp-4613-4621.

[22] E.Chun, L.Qin, L.Yong, S.MeiLin, Routing Protocols Overview and Design Issues for Self- Organized Network, Communication Technology Proceedings, 2000

[23] N. Razavi, M. R. Meybodi, Fair and Reliable Multicast Routing for Wireless Mesh Networks, IEEE Symposium on Computers \& Informatics, Kuala Lumpor, Malaysia, 2011, pp. $605-610$.

[24] X. Zhen, Bandwidth-satisfied Multi-path Multicast Routing in Wireless Mesh Network, International Conference on Computational Problem-Solving, 2010, pp. 300-305.

[25] F. Anwar, M. S. Azad, M. A. Rahman, S. M. S. Bari and M. H. Masud, Improved Link Repair Technique for Multicast Routing in Wireless Mesh Network, 4th IEEE International Conference on Mechatronics, Kuala Lumpur, Malaysia, 2011.

[26]M. Uma, Dr.G. Padmavathi, A comparative study and performance evaluation of reactive quality of service routing protocols in Mobile Adhoc networks, Journal of Theoretical and Applied Information Technology, vol. 6, 2009 , pp. $223-229$.

[27] J. Dong, R. Curtmola, and C.Nita-Rotaru, Secure HighThroughput Multicast Routing in Wireless Mesh Networks, IEEE Transactions on Mobile Computing, vol. 10, 2011, pp. 653-668.

[28] R. Gujral, Study and Comparison of Mesh and TreeBased Multicast Routing Protocols for MANETs, International Journal of Latest Trends in Engineering and Technology, vol. 1, July 2012, pp. 58 - 65 .

[29] W.A. Shittu, A-H.A. Hashim, F. Anwar, W. Al-Khateeb, A Proposed QoS Multicast Routing Framework for NextGeneration Wireless Mesh Network, IJCSNS International Journal of Computer Science and Network Security, vol.8, 2008, pp.280-284.

[30] S.J. Lee, W. Su, M. Gerla, On-demand multicast routing protocol in multihop wireless mobile networks, Mobile Networks and Applications, vol. 7, 2002, pp. 441-453.
[31] G. Lakshmikanth, S. Patel, A. Gaiwak, Performance Evaluation of Multicast Routing Protocols in MANET, International Journal of Recent Trends in Engineering, vol. 2, November 2009.

[32] S. Guo and O. Yang, Energy-aware multicasting in wireless ad-hoc networks: A survey and discussion, Computer Communications, vol. 30, June 2007, pp. 2129 -2148 .

[33] P. Nand, S.C. Sharma, Comparative study and Performance Analysis of FSR, ZRP and AODV Routing Protocols for MANET, 2nd International Conference and workshop on Emerging Trends in Technology (ICWET) 2011.

[34] Md. S. Azad, F. Anwar, Md. A.Rahman, A. H. Abdalla, A. U. Priantoro, O. Mahmoud, Performance Comparison of Proactive and Reactive Multicast Routing Protocols over Wireless Mesh Networks, International Journal of Computer Science and Network Security, vol.9, 2009.

[35] T.P. Singh, Neha,V.Das, Multicast Routing Protocols In Manets, International Journal of Advanced Research in Computer Science and Software Engineering, vol. 2, 2012.

[36] R. Gujral, S. Rana, A. Chaudhary, Study and Comparison of Mesh and Tree-Based Multicast Routing Protocols for MANETs, International Journal of Latest Trends in Engineering and Technology, vol. 1, 2012.

[37] K.A. Nair, G. Vidyadharan, P. Revathi, U. Devi, Analyzing the Performance of MAODV, ODMRP, MOSPF and PIM in Mobile Adhoc Networks, International Journal of Computer Science and Telecommunications, vol. 4, February 2013, pp. 24-29.

[38] S. J. Lee, W. Su, M. Gerla, On-Demand Multicast Routing Protocol in multihop wireless mobile networks, ACM/Kluwer Mobile Networks and Applications, vol. 7, 2002, pp. 441- 453

[39] K. Ritvanen, Multicast Routing and Addressing, HUT Report, Seminar on Internetworking, 2004.

[40] K. A. Nair, G. Vidyadharan T., P. Revathi,U.Devi G., Analyzing the Performance of MAODV, ODMRP, MOSPF and PIM in Mobile Adhoc Networks, International Journal of Computer Science and Telecommunications ,Vol. 4, 2013, pp. 24-29.

[41] M. S. Azad, F. Anwar, M. A. Rahman, A. H. Abdalla, A. U. Priantoro, O. Mahmoud, Performance Comparison of Proactive and Reactive Multicast Routing Protocols over Wireless Mesh Networks, International Journal of Computer Science and Network Security, Vol. 9, 2009 , pp. 55-62.

[42]K. Ritvanen, Multicast Routing and Addressing, Seminar on Internetworking, 2004.

[43] C. Perkins, E. Belding-Royer, S. Das, Ad hoc on-demand distance vector (AODV) routing, IETF RFC 3561, July 2003.

[44] P. Krishna, M. Subramanyam, K. Prasad, Mesh Based and Hybrid Multicast Routing Protocols for Manets: Current State of the Art, Global Journal of Computer Science and Technology, vol. 12, March 2012. 
[45] A. Spyropoulos, C.S. Raghavendra, Asymptotic capacitybounds for ad hoc networks revisited: the directionaland smart antenna cases, in: IEEE Global Telecommunications Conference (GLOBECOM), 2003, pp. 1216-1220.

[46] V. Srikanth, A. C. Jeevan, B. Avinash, T. S. kiran, S. S. Babu, A Review of Routing Protocols in Wireless Mesh Networks (WMN), International Journal of Computer Applications, Vol. 1, 2010, pp. 49-53.

[47] A. Le, D. Kum, and Y. Cho, An efficient Hybrid Routing Approach for Hybrid Wireless Mesh Networks, in: Proceedings of the 3rd international Conference and Workshops on Advances in information Security and Assurance, Seoul, Korea, June 2009,pp. 532 - 542.

[48] J. Sobczak, P. Zwierzykowski, Multicast Routing in Wireless Mesh Networks, the 8th Advanced International Conference on Telecommunications, 2012, pp. $62-68$.

[49] M. Mansouri, M. Meghdadi, R.A. Moghaddam, Achieving High-Dependability Optimal Multicast in Wireless Mesh Networks, IEEE, 2011, pp.609-613.

[50] O. B. Karimi, J.Liu, Z. Li, Multicast in Multi-channel Wireless Mesh Networks, IFIP Networking Conference, 2010, pp. 148-159.

[51] M. Jahanshahi, M. Dehghan, M. R. Meybodi, A mathematical formulation for joint channel assignment and multicast routing in multi-channel multi-radio wireless mesh networks, Journal of Network and Computer Applications, vol. 34, 2011, pp. 1869-1882.

[52]N. Kumar, N. Chilamkurti, J.H. Lee, A novel minimum delay maximum flow multicast algorithm to construct a multicast tree in wireless mesh networks, Computers and Mathematics with Applications, vol. 63, 2012, pp. 481491.

[53] L. Zhao, A. Al-Dubai, X. Liu , A new multicast routing algorithm for the Wireless Mesh Networks, 9th Annual Postgraduate Symposium on Convergence of Telecommunications, Networking and Broadcasting, Liverpool, 2008, pp. 151-156.

[54] D.T. Ahmed, S. Shirmohammadi, Architectural Analysis of Multicast Routing Protocols for Wireless Ad Hoc Networks, 6th International Conference on Networking, April 2007.

[55] Q, Xue, A, Ganz, Qos routing in mesh-based wireless networks, International Journal of Wireless Information Networks, vol. 9, July 2002, pp. 179-190.

[56] S. Keshav, R. Sharma, Issues and Trends in Router Design, IEEE Communications Magazine, May 1998.

[57] J. Dong, R. Burtmola, C. Nita-Rotaru, Secure HighThroughput Multicast Routing in Wireless Mesh Networks, Computer Science Technical Reports, IEEE Transactions On Mobile Computing, vol. 10, May 2011, pp. $653-668$.

[58] J.G. Jetcheva, D.B. Johnson, Adaptive Demand-Driven Multicast Routing in Multi-Hop Wireless Ad Hoc Networks, In Proceedings of the 2nd ACM international symposium on Mobile ad hoc networking \& computing, 2001, pp. 33-44.

[59] E.L. Madruga, J.J. Garcia-Luna-Aceves, Scalable Multicasting: The Core-Assisted Mesh Protocol, Mobile Networks and Applications 6, 2001, pp.151-165. 